\title{
Measurements of charmless $B$ decays at Belle
}

\author{
Min-Zu Wang ${ }^{* *}$ \\ Department of Physics, Institute of Astrophysics and \\ Leung Center for Cosmology and Particle Astrophysics, \\ National Taiwan University, Taiwan, R.O.C. \\ E-mail: mwang@phys.ntu.edu.tw
}

\begin{abstract}
Recent results of the charmless $B$ decays from Belle, namely the first inclusive mesurement of $B \rightarrow X_{s} \eta$ where $X_{s}$ stands for anything containing unit strangeness in the final states, are presented. These include the partial branching fraction as well as the differential braching fractions as a function of $M_{X_{s}}$ for $M_{X_{s}}<2.6 \mathrm{GeV} / c^{2}$, and the corresponding direct $\mathrm{CP}$ asymmetries. This study is based on a $605 \mathrm{fb}^{-1}$ data sample, consisting of 657 million $B \bar{B}$ pairs, collected by the Belle detector at the KEKB asymmetric energy $e^{+} e^{-}(3.5$ on $8 \mathrm{GeV})$ collider.
\end{abstract}

The XXXV International Conference on High Energy Physics,

July 22 - 282010

Paris, France

\footnotetext{
* Speaker.

$\dagger$ on behalf of the Belle Collaboration
} 


\section{Introduction}

Charmless $B$ decays are very interesting because there is no dominant $b \rightarrow c$ amplitude. It is quite possible that a large direct $\mathrm{CP}$ violation could be found due to the interference between decay amplitudes of comparable size, e.g. the $b \rightarrow s$ penguin and the $b \rightarrow u$ tree processes. It is also sensitive to new physics beyond the Standard Model through additional contributions from unknown heavy virtual particles in the $b \rightarrow s(d)$ penguin loop. Last but not the least, the information obtained from two-body charmless $B$ decays is very useful to determine the elements of the Cabibbo-Kobayashi-Maskawa (CKM) matrix and constrains the unitary triangle.

It is quite important to measure the inclusive process experimentally since the theoretical estimation of the corresponding $B$ decay branching fraction is more accurate due to less uncertainty introduced in the hadornization part. The first observation of the inclusive charmless hadronic $B$ decays, $B \rightarrow X_{s} \eta^{\prime}[1,2]$, revealed an unexpected contribution in the high $M_{X_{s}}$ region. The magnitude of the partial branching fraction is also difficult to explain theoretically. Some efforts have been made to explain this phenomenon, for example, the QCD anomaly model [3], a large intrinsic $c \bar{c}$ component of the $\eta^{\prime}$ [4] and new physics sources [5]. A recent development [6] based on the soft collinear effective theory indicates that the information from $B \rightarrow X_{s} \eta$ could be complementary and useful to understand the contribution from charming penguin amplitudes or higher order gluonic operators to both the $\eta$ and $\eta^{\prime}$ processes.

In this presentation, we will show the first inclusive measurement of $B \rightarrow X_{s} \eta$ [7]. The data sample used in this study is about $605 \mathrm{fb}^{-1}$ which contains $657 \times 10^{6} B \bar{B}$ pairs collected with the Belle detector [8] on the $\Upsilon(4 \mathrm{~S})$ resonance at the KEKB asymmetric energy $e^{+} e^{-}(3.5$ and $8 \mathrm{GeV})$ collider [9].

\section{Event selection and signal extraction}

We use a pseudo-inclusive method to reconstruct $B \rightarrow X_{s} \eta$ candidates, where $X_{s}$ consists of a $K^{+}$or $K_{S}^{0}$ and up to four pions, of which at most one can be a $\pi^{0}$. The event selection criteria for charged kaons and pions are based on the information obtained from the tracking system and the hadron identification system. $K_{S}^{0}$ candidates are reconstructed via the $\pi^{+} \pi^{-}$decay channel with an invariant mass close to its nominal mass [10]. The candidate must have a displaced vertex and flight direction consistent with a $K_{S}^{0}$ originating from the interaction point. $\eta$ and $\pi^{0}$ candidates are reconstructed by $\gamma \gamma$ with energies and positions determined by the electro-magnetic calorimeter and their momenta are recalculated by constraining the two photon invariant masses to the nominal ones [10]. Besides some minimum energy cuts on these $\gamma$ 's to reduce background, there is a special cut on the $\eta$ momentum in the center-of-mass $(\mathrm{CM})$ frame, $p_{\eta}^{C M}>2.0 \mathrm{GeV} / c$, which is useful to suppress secondary $\eta$ 's from $b \rightarrow c \rightarrow \eta$. We also reject candidate events when the final state particles can form $D^{0}, D^{+}, D_{s}^{+}, \eta_{c}$ or $\eta^{\prime}$.

The challenge of studying rare $B$ decays is the suppression of overwhelmingly large background from the continuum $e^{+} e^{-} \rightarrow q \bar{q}$ processes, where $q$ stands for $u, d, s$, or $c$ quark. One can use event-shape variables to separate the more spherically $B$ events from the jet-like continuum events. A GEANT [11] based Monte Carlo (MC) package is used to simulate signal events and estimate efficiencies in bins of $X_{S}$ mass, $M_{X_{s}}$. The MC samples consist of $B \rightarrow K \eta, B \rightarrow K^{*} \eta$, and 
$B \rightarrow X_{S} \eta$ components, where the $X_{S}$ mass spectrum is assumed to be flat and its fragmentation into hadrons is done via PYTHIA.

The collision kinematics at a B-factory provide an over-constrained system in which both the mass and energy of the $\mathrm{B}$ meson are known in the $\mathrm{CM}$ frame. We choose two kinematic variables in the $C M$ frame to identify the reconstructed $B$ meson candidates: the beam energy constrained mass $M_{\mathrm{bc}}=\sqrt{E_{\text {beam }}^{2}-p_{B}^{2}}$, and the energy difference $\Delta E=E_{B}-E_{\text {beam }}$, where $E_{\text {beam }}$ is the beam energy, and $p_{B}$ and $E_{B}$ are the momentum and energy, respectively, of the reconstructed $B$ meson. We use the $\Delta E$ information to select the best candidate if there are multiple candidates per event. We perform an unbinned extended likelihood fit on $M_{\mathrm{bc}}$ to determine the signal yield in $200 \mathrm{MeV} / \mathrm{c}^{2}$ bins of $M_{X_{S}}$ up to $2.6 \mathrm{GeV} / c^{2}$. The signal probability density function (PDF) is a Gaussian peaking at the $B$ meson mass. The continuum background is modeled by the ARGUS function [12]. There are non-negligible background events from $B \rightarrow X_{c} \rightarrow X_{s} \eta$ and its PDF can be described by a Gaussian peaking component and the ARGUS function. The background from rare $B$ decays like $B \rightarrow X_{s} \eta^{\prime}, B \rightarrow X_{s} \gamma$, and $B \rightarrow X_{d} \eta$ is estimated to be small. The expected yields are subtracted from the fit yields in order to get the final results.

\section{Results}

The fit to the $M_{\mathrm{bc}}$ spectrum with $M_{X_{s}}<2.6 \mathrm{GeV} / c^{2}$ is shown in Fig 1. There are about 1000 signal events observed after background subtraction. Its significance is $\sim 23$ and the significance is defined as $\sqrt{-2 \ln \left(L_{0} / L_{\max }\right)}$, where $L_{0}$ and $L_{\max }$ are the likelihood values returned by the fit with the signal yield fixed to zero and at its best fit value, respectively.We perform the same fitting procedure in $200 \mathrm{MeV} / c^{2}$ bins in $M_{X_{s}}$ to get the signal yields. After the efficiency correction, the differential branching fraction as a function of $M_{X_{s}}$ is shown in Fig. 2. There is a significant contribution from the high $M_{X_{S}}$ region. This feature is the same as that observed in $B \rightarrow X_{s} \eta^{\prime}$. We also check the direct CP asymmetry in bins of $M_{X_{s}}$ where the $B$ flavor is inferred from the charged kaon in the final state. There is no significant direct $\mathrm{CP}$ asymmetry observed. The overall value is $-0.13 \pm 0.04_{-0.03}^{+0.02}$ for $M_{X_{s}}<2.6 \mathrm{GeV} / c^{2}$ assuming the $B^{+} \rightarrow \pi^{+} \eta$ background contributes a CP asymmetry consistent with the existing world average [10].

\section{Acknowledgments}

The author wish to thank the KEKB accelerator group for the excellent operation of the KEKB accelerator. This work is supported by the National Science Council of the Republic of China under the grant NSC-98-2628-M-002-010.

\section{References}

[1] T.E. Browder et al. (CLEO Collaboration), Phys.Rev.Lett. 81, 1786 (1998).

[2] Throughout this report, inclusion of charge conjugate mode is always implied unless otherwise stated.

[3] D. Atwood and A. Soni, Phys.Lett.B 405, 150 (1997).

[4] I.E. Halperin and A. Zhitnitsky, Phys.Rev.Lett. 80, 438 (1998). 


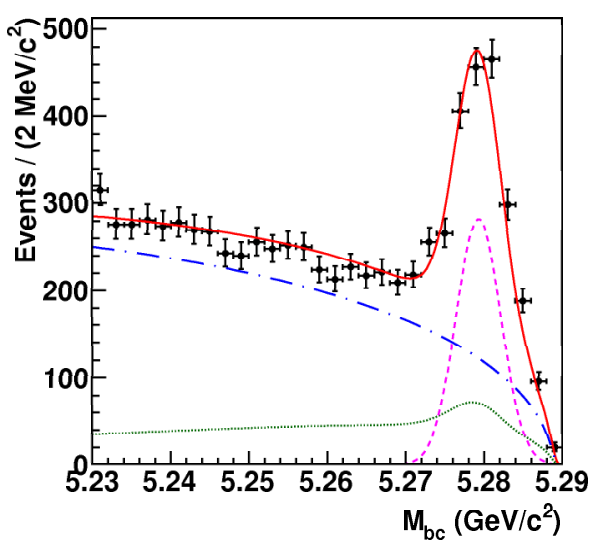

Figure 1: Distribution of $M_{\mathrm{bc}}$ for candidate events with $M_{X_{s}}<2.6 \mathrm{GeV} / c^{2}$. The solid curve represents the fit projection which is the sum of signal (Gaussian peak), continuum background (dash-dotted curve) and $b \rightarrow c$ background (dotted curve) estimations.

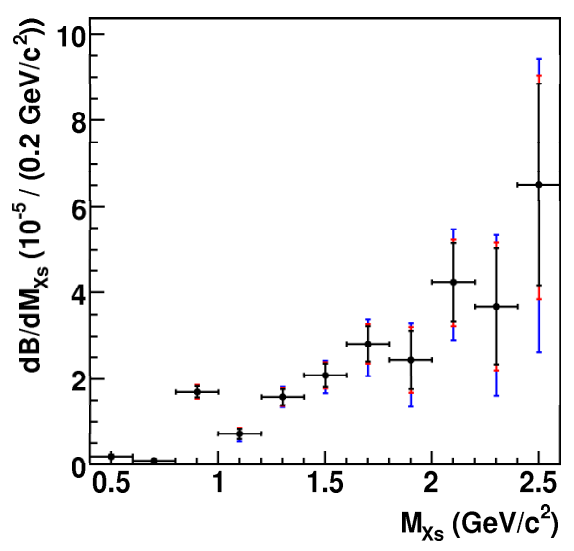

Figure 2: The differential branching fractions for $B \rightarrow X_{s} \eta$ as a function of $M_{X_{s}}$. The three error bars correspond to statistical error only, statistical plus systematic error, and total error.

[5] W.S. Hou and B. Tseng, Phys.Rev.Lett. 80, 434 (1998).

[6] J. Chay, C. Kim, A.K. Leibovich, and J. Zupan, Phys.Rev.D 76, 094031 (2007).

[7] K. Nishimura et al. (Belle Collaboration), Phys.Rev.Lett. 105, 191803 (2010) .

[8] A. Abashian et al. (Belle Collaboration), Nucl. Instr. and Meth. A479, 117 (2002).

[9] S. Kurokawa and E. Kikutani, Nucl. Instr. and Meth. A499, 1 (2003) and other papers included in this Volume.

[10] C. Amsler et al. (Particle Data Group), Phys. Lett. B667, 1 (2008).

[11] R. Brun et al., GEANT 3.21, CERN Report No. DD/EE/84-1 (1987).

[12] H. Albrecht et al. (ARGUS Collaboration), Phys. Lett. B 241, 278 (1990); ibid. B 254, 288 (1991). 